\title{
Podoplanin overexpression in human mesothelioma cell lines enhances the tumorigenic phenotype
}

\author{
EI YAMAKI, TOSHIKI YAJIMA, TAKAYUKI KOSAKA, AKIRA MOGI, \\ SHIGEBUMI TANAKA and HIROYUKI KUWANO
}

\author{
Department of General Surgical Science, Gunma University Graduate School of Medicine, Gunma, Japan
}

Received September 4, 2012; Accepted November 19, 2012

DOI: $10.3892 /$ or.2013.2225

\begin{abstract}
Podoplanin, a small type I integral membrane mucin-type sialoglycoprotein, serves as a useful marker for diagnosing malignant pleural mesothelioma (MPM); however, the physiological function of podoplanin in mesothelioma cells is not known. To elucidate the role of podoplanin in the pathogenesis of MPM, we generated two mesothelioma cell lines (PODO1 and PODO2) that stably express high levels of podoplanin. Although PODO1 cells proliferated to the same extent in culture or in nude mice, the survival rate of the mice was significantly reduced compared with that of the controls. We demonstrated that PODO1 and PODO2 cells had increased invasive ability in in vitro assays and induced upregulation of matrix metalloproteinase-1.PODO1 and PODO2 cultures could not be induced to undergo apoptosis when starved or treated with cis-diamminedichloroplatinum(II) (CDDP) compared with the controls. Moreover, silencing of podoplanin expression using RNA interference restored the ability of CDDP to induce apoptosis. Consistent with their growth properties, we detected constitutive activation of extracellular signal-regulated kinase in PODO1 and PODO2 cultures. These findings suggest that constitutive expression of podoplanin contributes to the invasive growth properties of mesothelioma cells and their resistance to apoptosis. Moreover, our data suggest that podoplanin or components of its signaling pathway, or both, may serve as important targets for developing novel treatments for MPM.
\end{abstract}

\section{Introduction}

Malignant pleural mesothelioma (MPM) is a highly aggressive tumor that arises from the mesothelial cells of pleural cavities. MPM is associated with asbestos and develops decades after

Correspondence to: Dr Ei Yamaki, Department of General Surgical Science, Gunma University Graduate School of Medicine, 3-39-22 Showa-machi, Maebashi, Gunma 371-8511, Japan

E-mail: eymk@showa.gunma-u.ac.jp

Key words: podoplanin, malignant pleural mesothelioma, matrix metalloproteinase-1, apoptosis, cis-diamminedichloroplatinum(II), extracellular signal-regulated kinase exposure. Epidemiological evidence indicates that the incidence of mesothelioma will increase over the next 10 to 20 years because of the historical use of asbestos and the quantities consumed (1). Since MPM responds poorly to conventional treatments, such as chemotherapy, surgery and radiation therapy, novel approaches for MPM treatment are urgently required. Therefore, it is critically important to define the molecular events involved in the induction and progression of MPM.

To address this issue, we focused our attention on the transmembrane type I glycoprotein called podoplanin, which consists of 162 amino acid residues, including 9 that reside in the cytoplasm. Podoplanin is widely expressed in the body and can be detected in kidney podocytes, placenta, skeletal muscle, lung, heart and mesothelial cells (2). Podoplanin null mice die at birth of lethal respiratory failure accompanied by immature lymphatic vessel formation $(3,4)$. Podoplanin is used as a specific marker for lymphatic endothelial cells (5). Although it is reasonable to assume that podoplanin plays an important role in the development of the lymphatic vascular system, its physiological function is unknown.

Podoplanin is expressed at abnormally high levels in many types of human cancers, including squamous cell carcinoma of the oral cavity, larynx, lung, cervix, esophagus and skin, as well as dysgerminomas of the ovary, granulosa cell tumors, and tumors of the central nervous system (6-10). Podoplanin is also expressed by MPM, and therefore is a useful marker for diagnosing this disease (11-16). Podoplanin expression also correlates with poor prognosis of oral, renal and brain tumors (17-19). Podoplanin promotes the migratory and invasive properties of the Madin-Darby canine kidney type II epithelial cell line (20).

Podoplanin, also known as Aggrus, possesses plateletaggregating activity associated with its PLAG domain. Moreover, podoplanin-induced platelet aggregation facilitates hematogenous metastasis. Chinese hamster ovary cell lines transfected with a podoplanin expression vector are more metastatic to lung than control cells, as determined using a mouse experimental metastasis model (21). Based on these findings, we believe that it is reasonable to conclude that podoplanin enhances the metastatic potential of tumor cells.

Here, to address the role of podoplanin in MPM and to determine whether it is a suitable target for designing more efficacious therapies for MPM, we generated an MPM cell line that overexpresses podoplanin. We determined the effects 
of overexpression of podoplanin in these cells in vitro with respect to their ability to migrate, invade an artifical matrix, and withstand the effects of inducers of apoptosis. The tumorigenic properties of the cells were assessed using a nude mouse xenograft model.

\section{Materials and methods}

Cell lines and animals. The MSTO-211H and NCI-H226 human mesothelioma cell lines were obtained from the American Type Culture Collection (ATCC, Manassas, VA, USA). The cells were maintained in RPMI-1640 medium (Sigma, St. Louis, MO, USA) containing $10 \%$ fetal bovine serum (FBS) and antibiotics $(100 \mathrm{U} / \mathrm{ml}$ penicillin and $100 \mu \mathrm{g} / \mathrm{ml}$ streptomycin). The medium was replaced every 2-3 days, and the cells were subcultured by treatment with $0.25 \%$ trypsin/0.53 mM EDTA.

Female BALB/c-nu/nu mice (age, 6 weeks) were obtained from Clea Japan Inc. (Tokyo, Japan) and housed in laminar flow cabinets under specific pathogen-free conditions. All experiments using mice were conducted in accordance with the guidelines of the National Institutes of Health (NIH, Bethesda, MD, USA) for the care and use of laboratory animals. The Animal Care and Experimentation Committee, Gunma University, approved the study protocol (approval no. 08-30).

Construction of a human podoplanin expression vector and stable transfection. A vector capable of expressing human podoplanin was constructed using the p3xFLAG-CMV-14 vector (Invitrogen, Tokyo, Japan) according to the manufacturer's instructions. The human podoplanin coding sequence was cloned using the pGEM $^{\circledR}-\mathrm{T}$ Easy vector (Invitrogen) into which we inserted the full-length podoplanin cDNA. The cDNA was generated by polymerase chain reaction (PCR) amplification using high-fidelity Platinum ${ }^{\circledR}$ TaqDNA polymerase (Invitrogen) and specific primers (forward, 5'-GGAAGGTGTCAGCTCTGCTC-3' and reverse, 5'-CGCC TTCCAAACCTGTAGTC-3') (6). The resulting construct was named p3xFLAG-Podoplanin-CMV-14. After verification by automated nucleotide sequence analysis, recombinant DNA plasmids were transfected into mesothelioma cells using Lipofectamine $^{\mathrm{TM}} 2000$ transfection reagent (Invitrogen) according to the manufacturer's instructions. The positive colonies were selected based on their resistance to $400 \mu \mathrm{g} / \mathrm{ml}$ Geneticin $^{\circledR}$ (Invitrogen) and identified by reverse transcriptase (RT)-PCR and flow cytometry. In the present study, clonal cell lines expressing podoplanin were designated PODO1 and PODO2. Cells transfected with the p3xFLAG-CMV-14 vector were designated $\mathrm{p} 3 \mathrm{x}$. The transfectants were used before passage 20 in all cases in order to minimize the potential impacts of clonal variations and phenotypic instability. Cell cultures were used for all functional and biological assays upon reaching 70-90\% confluence. The viability of cells in these cultures was $>95 \%$.

$R T-P C R$. Total RNA was extracted from cultured cells using TRIzol $^{\circledR}$ reagent (Invitrogen), and RT-PCR was performed according to the manufacturer's instructions using the following specific primers as described previously: matrix metalloproteinase-1 (MMP-1) (22) forward, 5'-GAGCAAACACATCTG
AGGTACAGGA-3' and reverse, 5'-TTGTCCCGATGATCT CCCCTGACA-3'; MMP-2 (22) forward, 5'-AGATCTTCTTC TTCAAGGACCGGTT-3' and reverse, 5'-GGCTGGTCAGT GGCTTGGGGTA-3'; MMP-7 (23) forward, 5'-AAACTCCC GCGTCATAGAAAT-3' and reverse, 5'-TCCCTAGACTGC TACCATCCG-3'; MMP-9 (24) forward, 5'-CACCTTCACTC GCGTGTAC-3' and reverse, 5'-CATCTGCGTTTCCAAACC GAG-3'; platelet-derived growth factor (PDGF-BB) (25) forward, 5'-CTAGGCTCCAAGGGTCTCCT-3' and reverse, 5'-GAGAAAGATCGAGATTGTGC-3'; PDGFR- $\beta$ (26) forward, 5'-GTGAACGCAGTGCAGACTGT-3' and reverse, 5'-AGGTGTAGGTCCCCGAGTCT-3'. As internal controls, glyceraldehyde-3-phosphate dehydrogenase (GAPDH) (27) forward, 5'-CGACCACTTTGTCAAGCTCAT-3' and reverse, 5'-CCCTGTTGCTGTAGCCAAATT-3' and $\beta$-actin (27) forward, 5'-GATGATGATATCGCCGCGCT-3' and reverse, 5'-TGGGTCATCTTCTCGCGGTT-3' were used.

Flow cytometry. The transfected p3x, PODO1 and PODO2 cells were harvested, and single-cell suspensions ( $1 \times 10^{6}$ cells) were prepared. The cells were treated according to the standardized protocol as follows. The cells were harvested after brief exposure to trypsin. After washing with phosphate-buffered saline (PBS), the cells were treated with anti-podoplanin mouse monoclonal antibody (ab10288; Abcam, Tokyo, Japan) for $30 \mathrm{~min}$ at $4^{\circ} \mathrm{C}$ and then incubated with FITC-conjugated anti-mouse immunoglobulin antibody (DakoCytomation, Glostrup, Denmark) for $30 \mathrm{~min}$ at $4^{\circ} \mathrm{C}$. Flow cytometric analysis was performed using a BD FACSCalibur flow cytometer (Becton-Dickinson, San Jose, CA, USA), and the data were analyzed using CellQuest software (Becton-Dickinson).

Platelet aggregation assay. Platelet aggregation was measured using a platelet aggregometer, PA-200 (Kowa Co., Ltd., Nagoya, Japan). Platelet-rich plasma (PRP) and platelet-poor plasma (PPP) were obtained from the blood of healthy donors, which was treated with $3.13 \%$ sodium citrate (blood:sodium citrate, 9:1) to inhibit coagulation and then centrifuged sequentially at $170 \mathrm{x} \mathrm{g}$ for $10 \mathrm{~min}$ and $1,880 \mathrm{x} \mathrm{g}$ for $10 \mathrm{~min}$. When the platelet count of PRP was $>200 \times 10^{9} / 1$, PRP was diluted by PPP to $200 \times 10^{9} / 1$. The sample was placed in a plastic cuvette containing a magnetic stirring bar at $37^{\circ} \mathrm{C}$ for $10 \mathrm{~min} . \mathrm{CaCl}_{2}$ (200 mM) was added, and $3 \mathrm{~min}$ later, platelet aggregation was initiated by adding PODO1, PODO2, or p3x cells $\left(5 \times 10^{6}\right.$ cells/ sample), and the samples were monitored for at least $15 \mathrm{~min}$.

Cell migration and Matrigel invasion assay. Cell invasiveness was assessed using BioCoat ${ }^{\mathrm{TM}}$ Matrigel $^{\mathrm{TM}}$ invasion chambers (Becton-Dickinson) according to the protocol provided by the manufacturer. In brief, $1 \times 10^{4}$ cells were seeded in the upper compartment in serum-free RPMI-1640 medium. The lower compartment was filled with RPMI-1640 medium containing $10 \% \mathrm{FBS}$ as a chemoattractant. After incubation for $48 \mathrm{~h}$ at $37^{\circ} \mathrm{C}$ in humidified air containing $5 \% \mathrm{CO}_{2}$, non-invading cells remaining on the upper surface of the chamber were removed by scrubbing with a cotton-tipped swab. The invading cells that adhered to the bottom surface of the chamber membrane were fixed and counted after staining with Mayer's hematoxylin and eosin. The assays were performed in triplicate, at least 5 fields were counted/filter, and mean cell numbers and 
standard deviations were calculated. For the cell migration analysis, polycarbonate filters (pore size, $6 \mu \mathrm{m}$ ) were used without Matrigel (BD Falcon ${ }^{\mathrm{TM}}$ Cell Culture Inserts; BectonDickinson).

Wound migration assay. Mesothelioma cell lines were seeded in a Petri dish at $1 \times 10^{6}$ cells $/ \mathrm{ml}$ and grown in RPMI-1640 medium containing 10\% FBS until the cells were almost confluent. The medium was removed and two lines were drawn using a pipette tip through the cell monolayer. The cells were washed twice with PBS and RPMI-1640 medium. After $0,6,12$ and $24 \mathrm{~h}$ images were captured using a Nikon TMS microscope at magnification, $\mathrm{x} 40$.

Cell proliferation assay. Cell Counting Kit (CCK)-8 (Dojindo, Tokyo, Japan) was used to determine cell proliferation. The cells were harvested and deposited in 96-well plates $\left(1 \times 10^{4}\right.$ cells/well) and maintained in a humidified atmosphere containing $5 \% \mathrm{CO}_{2}$ at $37^{\circ} \mathrm{C}$. At each time point, $10 \mu \mathrm{l}$ of the cell counting solution was added to triplicate wells and incubated for $0.5 \mathrm{~h}$. The formazan dye generated by dehydrogenases in the cells was dissolved in $100 \mu \mathrm{l} /$ well $1 \mathrm{~N} \mathrm{HCl}(100 \mu \mathrm{l} /$ well $)$, and the absorbance at $450 \mathrm{~nm}$ was measured using a microtiter plate reader (Molecular Devices, Sunnyvale, CA, USA) to calculate the numbers of viable cells in each well.

Cell extraction and western blot analysis. Lysates were prepared from exponentially growing cells using a buffer containing the following components: $20 \mathrm{mM}$ Tris- $\mathrm{HCl}$ (pH 7.6), 1 mM EDTA, $140 \mathrm{mM} \mathrm{NaCl}, 1 \%$ Nonidet P-40, $1 \%$ aprotinin, $1 \mathrm{mM}$ phenylmethylsulfonyl fluoride, and $1 \mathrm{mM}$ sodium vanadate. Protein concentrations were determined using a BCA protein assay kit (Pierce, Rockford, IL, USA). Protein $(40 \mu \mathrm{g})$ samples from each cell line were added to sodium dodecyl sulphate (SDS) sample buffer [100 mM Tris- $\mathrm{HCl}$ (pH 8.8), $0.01 \%$ bromophenol blue, $36 \%$ glycerol, $4 \%$ SDS and $1 \mathrm{mM}$ dithiothreitol], boiled for $5 \mathrm{~min}$, and electrophoresed through a 5-20\% acrylamide gel (TrisTricine Ready Gels; Bio-Rad, Tokyo, Japan). Proteins were electrophoretically transferred to a Hybond-N+ nitrocellulose membrane (Amersham Pharmacia Biotech, Buckinghamshire, UK). Antibodies to PKB/Akt (BD Biosciences, Tokyo, Japan) p44/p42 mitogen-activated protein kinase (MAPK), p38 MAPK and SAPK/JNK (MAPK family antibody sampler kit, phospho-Akt, phospho-p44/p42 MAPK, phospho-p38 MAPK, and phospho-SAPK/JNK (phospho-MAPK family antibody sampler kit) (all were from Cell Signaling Technology, Inc., Danvers, MA, USA) and caspase-3 (Santa Cruz Biotechnology, Inc., Santa Cruz, CA, USA) were used. The antibodies were diluted $(1: 1,000)$ and incubated with the membranes overnight at $4^{\circ} \mathrm{C}$. After washing, the proteins were incubated with the secondary antibody (horseradish peroxidase-conjugated antimouse or anti-rabbit immunoglobulin $\mathrm{G}$ antibody) diluted 1:1,000. The antigen-antibody complexes were detected using an enhanced chemiluminescence detection system (Amersham Pharmacia Biotech). Membranes were reprobed using anti$\beta$-actin (Sigma) as a loading control.

Assays for apoptosis. Mesothelioma cell lines were seeded in complete growth medium, incubated for $16 \mathrm{~h}$, and treated with cis-diamminedichloroplatinum(II) (CDDP) (cisplatin) for $48 \mathrm{~h}$ at $80 \%$ confluence. The MSTO-211H and NCI-H226 cultures were treated with $5 \mu \mathrm{M}\left(\mathrm{IC}_{50}\right)$ and $25 \mu \mathrm{M}$ CDDP. $\mathrm{CDDP}$ was not added to the control cells. The $\mathrm{IC}_{50}$ value is defined as the concentration required to reduce absorbance (calculated from the growth curve) by $50 \%$. After $48 \mathrm{~h}$, detached cells were collected, and the adherent cells were harvested after treatment with trypsin. The cells $\left(1 \times 10^{5}\right)$ were washed twice with PBS and resuspended in $250 \mu \mathrm{l}$ of binding buffer (Annexin V/FITC Apoptosis Detection kit; Sigma) that contained $2 \mu \mathrm{l}$ of $20 \mathrm{mg} / \mathrm{ml}$ propidium iodide (PI) and $2.5 \mu \mathrm{l}$ of Annexin V/FITC. The data were collected using FACSCalibur (Becton-Dickinson). FITC and PI emissions were detected in the FL-1 and FL-2 channels, respectively.

An Active Caspase-3 Apoptosis kit I (BD Biosciences) was used to detect the direct activation of caspase-3 according to the manufacturer's instructions. In brief, the cells $\left(1 \times 10^{5}\right)$ were washed twice with cold PBS, resuspended in Cytofix/Cytoperm solution for $20 \mathrm{~min}$, and washed twice with Perm/Wash buffer. The cells were then resuspended in the FITC-conjugated active caspase- 3 monoclonal antibody and incubated for $30 \mathrm{~min}$. Subsequently, the cell suspension was washed twice with Perm/Wash buffer and analyzed using a flow cytometer.

Gene silencing. We synthesized 21-nucleotide-long small interfering RNAs (siRNAs). Stealth RNAi ${ }^{\mathrm{TM}}$ siRNAs were obtained from Invitrogen. The podoplanin siRNA target sequences synthesized in our laboratory were as follows (4): (R1) 5'-GCG AAGACCGCUAUAAGUCdTdT-3' and (R2) 5'-AAGAUG GUUUGUCAACAGUdTdT-3'. NCI-H226 cultures were transfected with various concentrations (50-1,000 pmol) of siRNAs or with equimolar concentrations of the control vector using Lipofectamine $^{\mathrm{TM}} 2000$ transfection reagent according to the manufacturer's instructions. The cells were harvested $48 \mathrm{~h}$ after transfection, and the podoplanin expression level was confirmed by flow cytometry. CDDP was added, and $48 \mathrm{~h}$ after this treatment, the apoptosis detection assay was performed.

Mouse model of mesothelioma. PODO1 cells $\left(1 \times 10^{7}\right)$ in $0.2 \mathrm{ml}$ complete medium were injected into the dorsum of female BALB/c-nu/nu mice. Tumor volumes were calculated using the formula (long axis $\mathrm{x}$ short axis $\mathrm{x}$ short axis)/2. The growth curves were generated by considering the mean tumor volume at different time points.

Statistical analysis. Unpaired Student's t-test and one-factor analysis of variance (Scheffe's post hoc test) were used to determine statistical significance. Survival rates were calculated by the Kaplan-Meier method, and the log-rank test was used to assess the differences in prognosis between two groups. Differences were considered significant at p-values of $<0.05$. Statistical analyses were performed using StatView software (version 5; SAS Institute, Cary, NC, USA).

\section{Results}

Generation of mesothelioma cell lines that stably express human podoplanin. To investigate the potential role of podoplanin in the pathogenesis of MPM, we cloned podoplanin cDNA into an expression vector and succeeded in estab- 
A

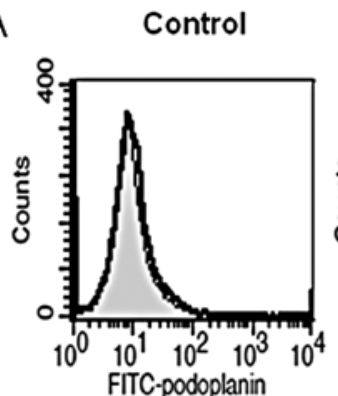

B

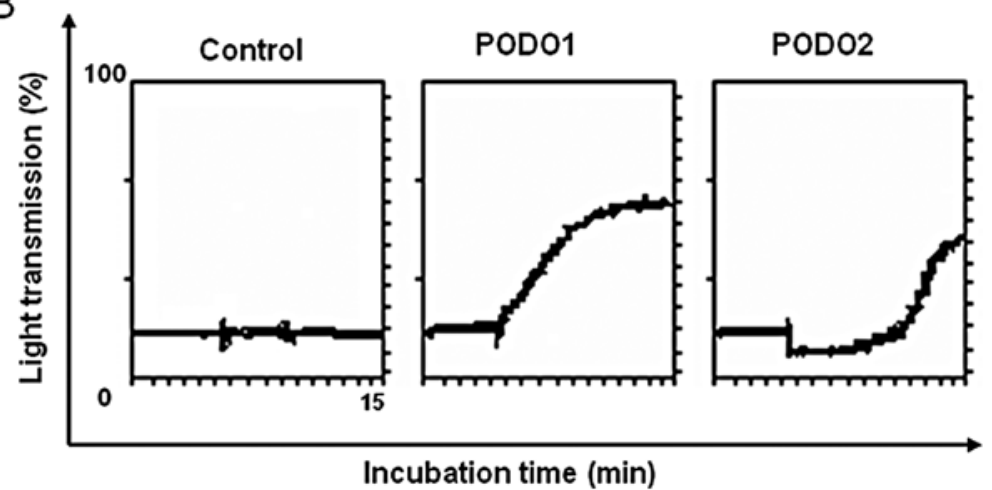

Figure 1. Comparison of podoplanin expression in mesothelioma cell lines. Podoplanin expression was evaluated by flow cytometry. (A) Analysis of podoplanin expression in mesothelioma cell lines (PODO1 and PODO2) transfected with a podoplanin expression vector. Podoplanin expression was undetectable in cells transfected with p3x. In contrast, PODO1 and PODO2 expressed high levels of podoplanin. (B) The platelet aggregation activity of each clone was estimated by incubating the clone with diluted human platelets as described in Materials and methods.

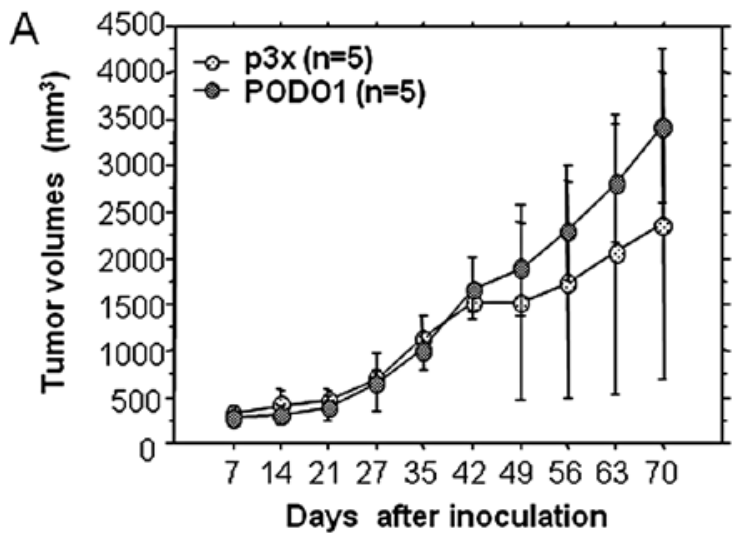

$\mathrm{B}$

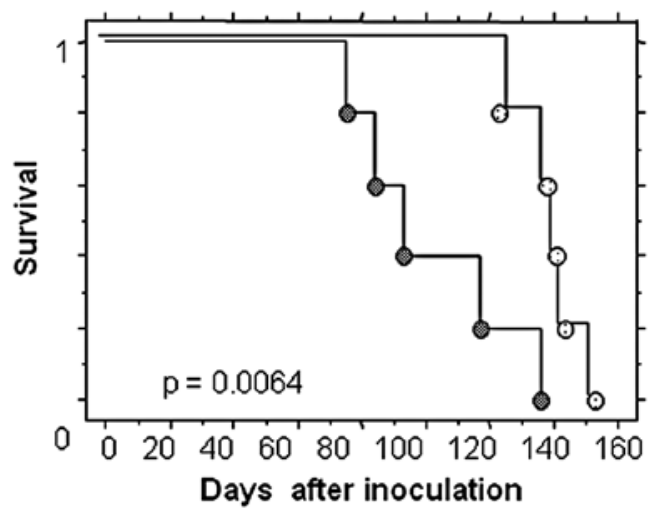

Figure 2. PODO1 and $\mathrm{p} 3 \mathrm{x}$ cells were inoculated subcutaneously into nude mice. (A) Tumor volume was measured using calipers at the times indicated. Data represent means \pm SEM. (B) The survival rate of mice bearing tumors induced by $\mathrm{p} 3 \mathrm{x}$ and PODO1 was evaluated by the Kaplan-Meier method and the log-rank test. lishing mesothelioma cell lines that stably expressed human podoplanin. We chose to utilize the MSTO-211H cell line, which was established from the pleural effusion of a patient with biphasic mesothelioma of the lung and does not detectably express endogenous podoplanin as assessed by RT-PCR and flow cytometry (Fig. 1A) (data not shown). This cell line forms tumors in nude mice (28) and consists of biphasic-type mesothelioma cells, which exhibit features of epithelial and sarcomatous cells. Flow cytometry revealed that podoplanin expression levels in two clones of podoplanin transfectants (PODO1 and PODO2) were significantly higher than those in the cells transfected with the empty vector (p3x). These two clones were used in subsequent experiments. Podoplanin induces platelet aggregation, which we confirmed here using the aggregometer. Platelet aggregation was detected only when the podoplanin transfectants were included in the in vitro aggregation assay (Fig. 1B).

Tumors formed by the podoplanin transfectant reduced the survival of nude mice. We assessed the tumorigenic properties of PODO1 by injecting the cells into nude mice and compared tumor size and survival rates with mice inoculated with a culture of $\mathrm{p} 3 \mathrm{x}$. The survival rate of mice inoculated with PODO1 was significantly lower than that of mice inoculated with p3x (Fig. 2). We did not observe the presence of metastases to other organs, including the lung. No significant difference was observed between the growth of the podoplanin transfectant compared with $\mathrm{p} 3 \mathrm{x}$ for $42 \mathrm{~h}$ after inoculation. After this duration, the central mass of the tumor necrotized in the control mice, but the tumor volume did not change. These results suggest that podoplanin expression did not contribute to 

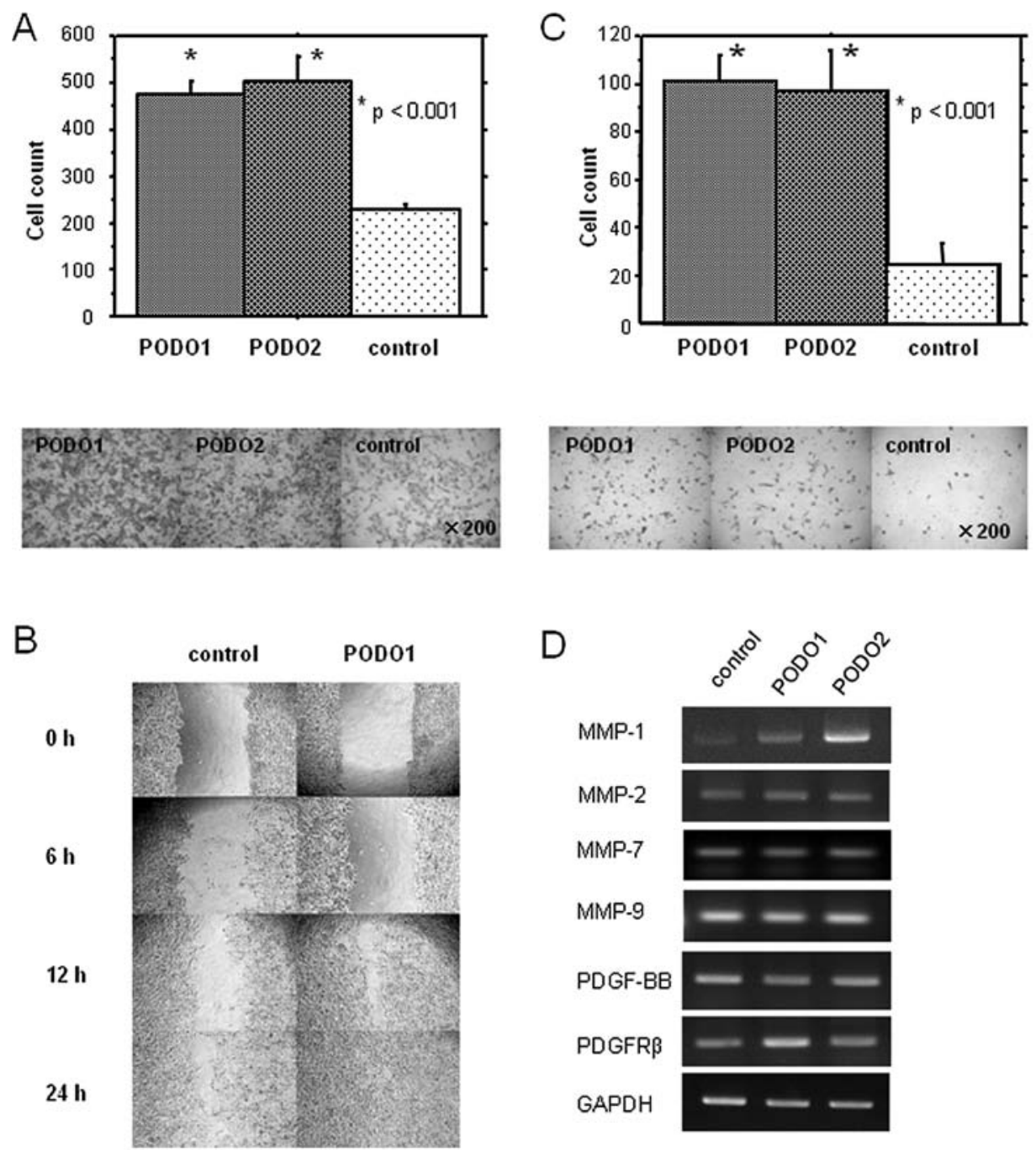

Figure 3. Enforced expression of podoplanin enhances cell motility and invasiveness. (A) Histogram showing chemotaxis by PODO1, PODO2 and p3x cells. Data from three independent experiments are summarized. (B) Wound closure in monolayers of PODO1 and p3x cells over $24 \mathrm{~h}$. (C) Cell invasion was analyzed using a cell chamber (pore size, $6 \mu \mathrm{m}$ ) coated with Matrigel (BioCoat ${ }^{\mathrm{TM}}$ Matrigel ${ }^{\mathrm{TM}}$ invasion chambers). The cells were counted in three fields of the membrane, and the data from three independent assays are summarized. (D) RT-PCR analyses showing the expression levels of endogenous MMP-1, -2, -7, -9 , PDGF-BB, PDGFR- $\beta$ and GAPDH mRNA in PODO1 and PODO2 cells. GAPDH was used as an internal control.

the growth of mesothelioma cell lines in nude mice. Therefore, podoplanin expression reduced the survival of the mice but did not affect the growth potential of the transfectants.

Podoplanin promotes migration and invasive properties of mesothelioma cell lines in vitro. Evidence suggests that podoplanin expression is associated with the enhanced ability of cancer cells to migrate $(10,20)$. We, therefore, performed in vitro motility assays to determine whether our podoplanin transfectants possessed this property. PODO1 and PODO2 cells exhibited higher migratory activities than $\mathrm{p} 3 \mathrm{x}(\mathrm{p}<0.05)$ (Fig. 3A).

To confirm and extend these findings, we performed an in vitro wound-healing assay. As shown in Fig. 3B, PODO1 exhibited increased cell migration to a greater extent than $\mathrm{p} 3 \mathrm{x}$. Moreover, the abilities of PODO1 and PODO2 cells to invade a layer of Matrigel were also significantly enhanced compared with those of $\mathrm{p} 3 \mathrm{x}$ cells (Fig. 3C). These results suggest that podoplanin overexpression promotes the migration and invasion of mesothelioma cells.
Members of the MMP family play a crucial role in the migration and invasiveness of cancer cells. Signaling by the PDGF-BB $(25,29)$ isoform through its receptor, PDGFR- $\beta$, also promotes the migration and invasiveness of mesothelioma cells. Therefore, we examined the expression of MMP-1, -2, -7, -9 and PDGF in the podoplanin transfectants. We found that the expression levels of MMP-1 mRNA, but not of MMP-2, -7 and -9 mRNA, were increased in PODO1 and PODO2 compared with p3x cells (Fig. 3D). No differences were observed in the expression levels of PDGF-BB and PDGFR- $\beta$ mRNA between PODO1 and PODO2 compared with $\mathrm{p} 3 \mathrm{x}$ cells. These results suggest that podoplanin promotes tumor cell invasion in vitro via upregulation of MMP-1 expression.

Podoplanin overexpression enhances cell proliferation and attenuates induction of apoptosis. We assessed the proliferative capacity of the podoplanin transfectants using an MTT assay. No significant differences were observed between the proliferative capacities of the two podoplanin transfectants and $\mathrm{p} 3 \mathrm{x}$ after $24 \mathrm{~h}$, indicating that podoplanin expression was 
A

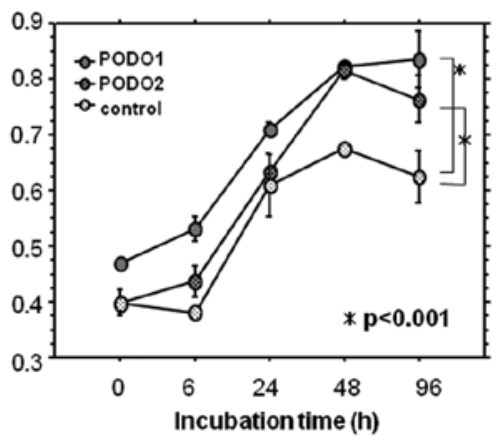

$B$
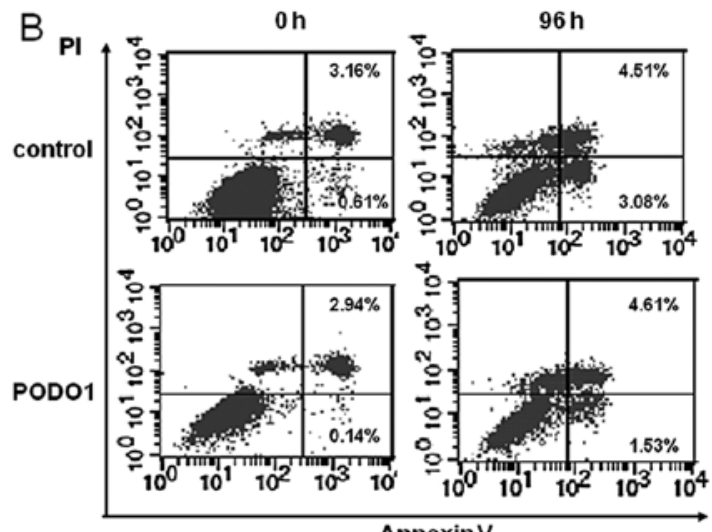

Annexin V

Figure 4. Proliferation and apoptosis assays. (A) The proliferation of PODO1 and POD2 cells was determined using a cell counting kit based on formazan formation ( $\mathrm{p}<0.001)$. (B) Annexin V/propidium iodide (PI) staining of PODO1 cells.

A

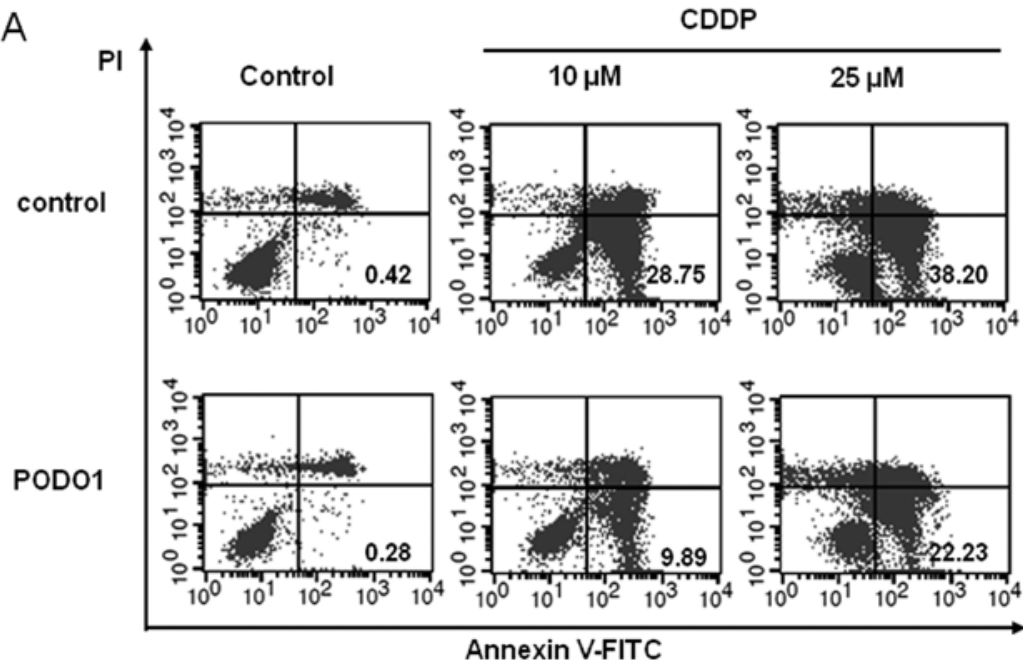

B

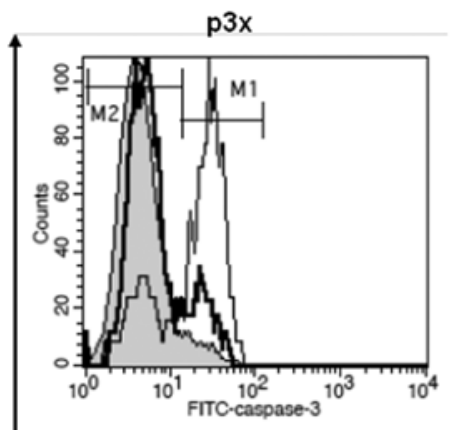

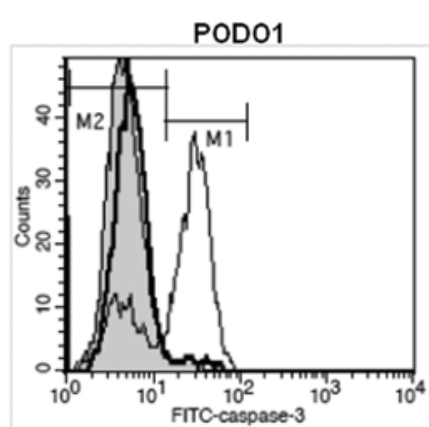

Active caspase-3

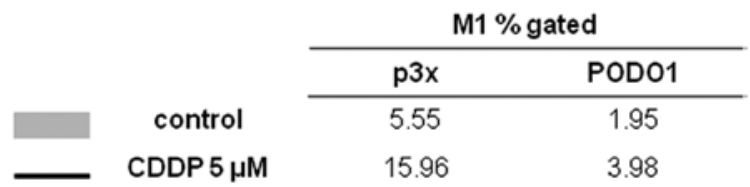

Figure 5. Podoplanin expression inhibits CDDP-induced apoptosis. (A) Annexin V/propidium iodide (PI) assay. PODO1 and p3x cultures were treated with CDDP [ $\left(5 \mu \mathrm{M}\left(\mathrm{IC}_{50}\right)\right.$ and $\left.\left.25 \mu \mathrm{M}\right)\right]$ for $48 \mathrm{~h}$. CDDP was not added to the control culture. (B) Caspase-3 activation in p3x and PODO1 cultures was evaluated using the sample described in Fig. 6A.

dispensable for the proliferation of mesothelioma cell lines in vitro during this period (Fig. 4A). However, the proliferative capacities of the podoplanin transfectants after 48 and $96 \mathrm{~h}$ were significantly greater than those of $\mathrm{p} 3 \mathrm{x}(\mathrm{p}<0.001)$.
Cell number is regulated by the balance between cell proliferation and apoptosis, and this raised the possibility that the abilities of the podoplanin transfectants to undergo apoptosis may have been altered after $48-96 \mathrm{~h}$ in culture. Therefore, 


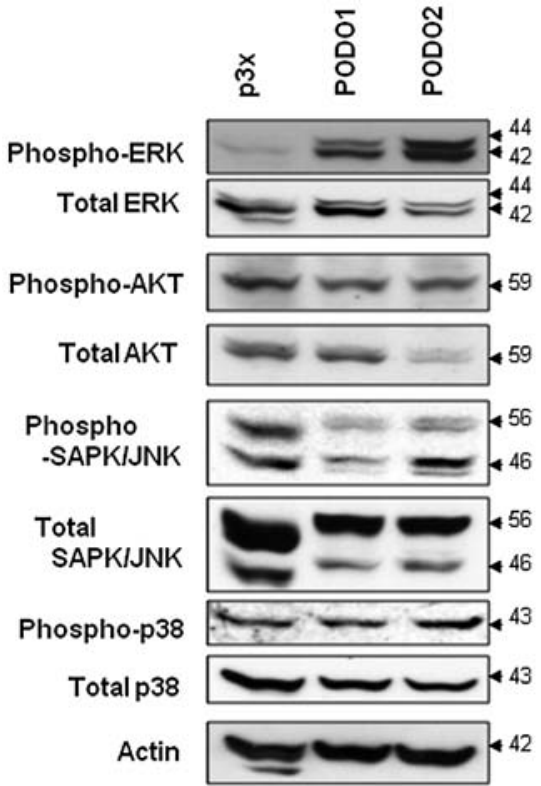

Figure 6. Western blot analysis of ERK signaling pathway components. Cell lysates prepared from PODO1, PODO2 and $\mathrm{p} 3 \mathrm{x}$ cultures were analyzed. $\beta$-actin was used as a loading control.

we next examined apoptosis in the transfectants after $96 \mathrm{~h}$ of culture. Twice the number of apoptotic cells was present in the $\mathrm{p} 3 \mathrm{x}$ cultures compared with the PODO1 cultures (Fig. 4B). These results indicate that podoplanin overexpression inhibited serum starvation-induced apoptosis.

Podoplanin inhibits induction of apoptosis by CDDP. We next determined whether podoplanin overexpression inhibited the induction of apoptosis by CDDP (cisplatin), a drug widely used to treat mesothelioma. We determined the induction of apoptosis $48 \mathrm{~h}$ after CDDP treatment. The number of apoptotic cells in the PODO1 cultures was reduced compared with that in the $\mathrm{p} 3 \mathrm{x}$ cultures (Fig. 5A). This effect was confirmed by determining active caspase expression (Fig. 5B). Taken together, these results suggest that podoplanin overexpression (in a mesothelioma cell line) plays an important role in inhibiting apoptosis induced by serum starvation and CDDP.

Podoplanin overexpression induces ERK phosphorylation in mesothelioma cells. To identify the molecular mechanism responsible for the anti-apoptotic effect of podoplanin overexpression in mesothelioma cells, we examined the activation of the ERK signaling pathway. The phosphorylation levels of JNK, p38 and AKT did not differ between PODO1 and PODO2 compared with p3x cells (Fig. 6). In contrast, the phosphorylation level of ERK was dramatically enhanced in the two podoplanin transfectants. Thus, podoplanin-induced ERK activation may be inhibited by starvation-induced apoptosis in mesothelioma cells.

Silencing of the gene encoding podoplanin induces apoptosis in mesothelioma cells that express high levels of endogenous podoplanin. To confirm that podoplanin overexpression affects apoptosis, we studied a mesothelioma cell line that expresses high levels of endogenous podoplanin (NCI-H226). A siRNA targeted to the mRNA encoding podoplanin significantly
A

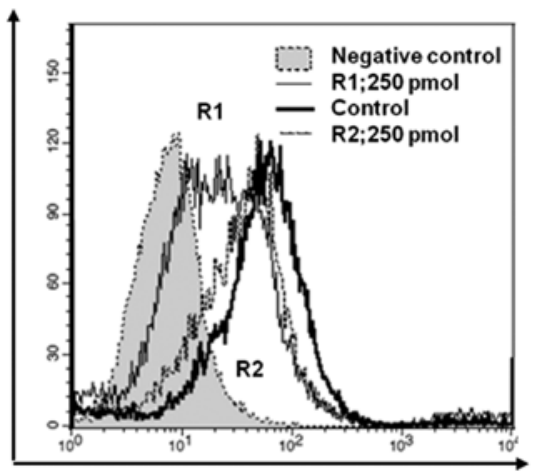

B

FITC-podoplanin

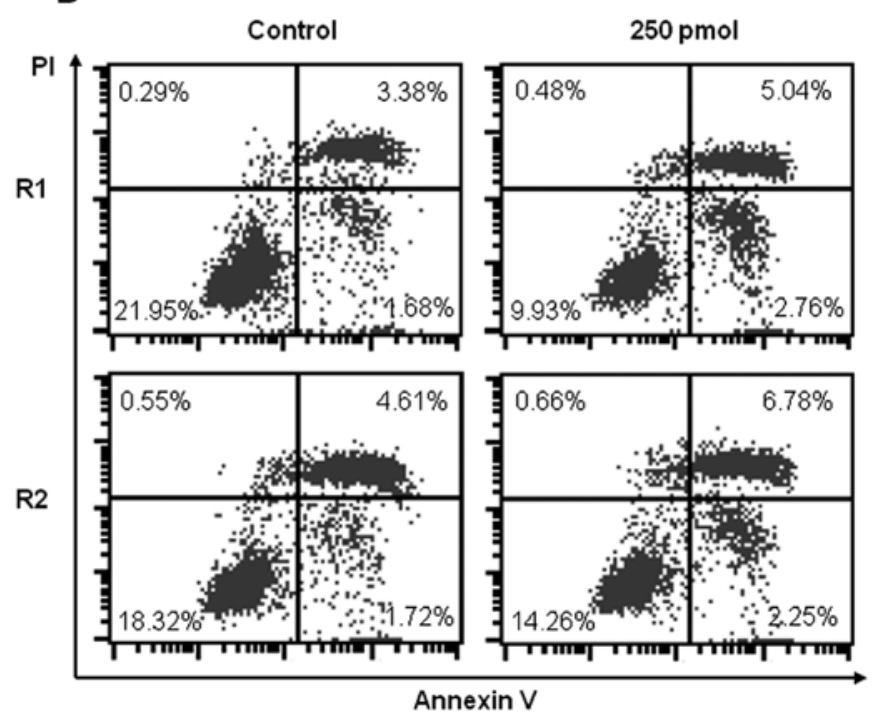

Figure 7. (A) NCI-226 cells were transfected with a podoplanin siRNA (50-1,000 pmol) or with equimolar concentrations of the control vector and were analyzed using flow cytometry. The cells were harvested $48 \mathrm{~h}$ after transfection. (B) CDDP was added, and $48 \mathrm{~h}$ after this treatment, the apoptosis detection assay was performed.

reduced the expression level of endogenous podoplanin after $48 \mathrm{~h}$ (Fig. 7A). The proportion of apoptotic cells was slightly but significantly increased in the podoplanin transfectant compared with the control. Apoptosis was detected $48 \mathrm{~h}$ after CDDP treatment (Fig. 7B).

\section{Discussion}

In the present study, we examined the potential role of podoplanin in the pathophysiological properties of MPM using mesothelioma cell lines. We found that the survival rate of nude mice inoculated with a mesothelioma cell line that stably overexpressed podoplanin (PODO1) was significantly reduced compared with the survival of mice inoculated with the same cell line transfected with the empty expression vector ( $\mathrm{p} 3 \mathrm{x}$ ). Podoplanin overexpression in this mesothelioma cell line augmented motility and invasive properties in vitro. Moreover, podoplanin overexpression imparted resistance to apoptosis, and reduction of podoplanin expression using RNA interference led to increased apoptosis in vitro. These results suggest that podoplanin plays an important role in the pathogenesis of MPM. 
The physiological function of podoplanin is unknown. We found that the survival of nude mice inoculated with PODO1 cells was reduced compared with that of mice inoculated with p3x. However, tumor growth induced by PODO1 was comparable with that induced by $\mathrm{p} 3 \mathrm{x}$ at an early stage $(24 \mathrm{~h})$ after inoculation. A significant difference in survival was observed at 48 and $96 \mathrm{~h}$. However, podoplanin, also called Aggrus, induces platelet aggregation, and this activity is associated with its PLAG domain. Platelet aggregation protects tumor cells from immunological assault in the circulation and facilitates the formation of hematogenous metastases (30). Podoplanin-transfected $\mathrm{CHO}$ cells were found to increase the number of metastases to the lung in an experimental mouse model of metastatic growth (21). Therefore, podoplanin may enhance the metastatic potential of mesothelioma cell lines in nude mice by promoting platelet aggregation. However, in the present study, we did not detect obvious metastatic lesions (data not shown), indicating that another mechanism is responsible for the significantly poorer survival rates of nude mice inoculated with PODO1 when compared with mice inoculated with p3x cells.

Podoplanin overexpression increases cell migration and invasion of the MCF7 breast cancer and HaCaT keratinocyte cell lines $(10,20,31)$. Consistent with these findings, we found that podoplanin overexpression in a mesothelioma cell line increased its in vitro invasive and migratory potential. Therefore, podoplanin may play a critical role in the motility and invasion of cancer cells.

There are several possible explanations that account for how podoplanin expression serves to induce the motility and invasive property of the mesothelioma cell line studied here. Podoplanin complexed with CLEC-2 on platelets enhanced the release of PDGF from cancer cells (32). PDGF enhanced cell invasion and motility of a mesothelioma cell line in vitro (25). This supports the first possibility that the induction of PDGF expression by podoplanin may affect the motility and invasiveness of mesothelioma cells. However, we found that podoplanin overexpression did not affect the expression of PDGF mRNA, making this possibility unlikely.

MMPs, in particular MMP-2 and MMP-9, are expressed by most cancer cells, including MPM, and they play a key role in influencing cell motility and invasiveness (33). Treatment of podoplanin-transfected breast cancer cell lines with tissue inhibitor of MMPs (TIMP2), a general MMP inhibitor, almost completely abrogates cellular invasiveness (10). Therefore, taken together with our present findings, it is also possible that MMP-1 is induced by podoplanin and acts to enhance the motility and invasiveness of mesothelioma cell lines.

Another notable finding of the present study is that podoplanin overexpression inhibited the induction of apoptosis by starvation and the chemotherapeutic drug CDDP (cisplatin). Two major apoptosis pathways are initiated by cell-surface proteins of the TNFR family, while other pathways are initiated by stress signals $(34,35)$. MAPK cascades are essential components of the latter apoptotic signaling pathways. In the present study, we found that podoplanin overexpression led to constitutive activation of ERK in PODO1 and PODO2 cells. Moreover, the activity of the MEK-ERK signaling pathway reduces sensitivity of ovarian carcinoma cells to CDDP (36). Taken together, these findings indicate that diminished levels of podoplanin increased the sensitivity of malignant mesothelioma cells to CDDP by downregulating ERK activation.

In summary, we discovered a new functional role of podoplanin in mesothelioma cell lines. Podoplanin overexpression led to anti-apoptotic effects and increased tumor malignancy in vitro and in vivo. Moreover, silencing the expression of the gene encoding podoplanin increased the number of cells undergoing apoptosis. These findings lead us to conclude that podoplanin may serve as an important target for developing more efficacious treatments for MPM.

\section{Acknowledgements}

We thank Dr Hideki Uchiumi (Department of Medicine and Clinical Science, Gunma University) for the excellent technical assistance in the platelet aggregation analysis.

\section{References}

1. Fennell DA, Gaudino G, O'Byrne KJ, Mutti L and van Meerbeeck J: Advances in the systemic therapy of malignant pleural mesothelioma. Nat Clin Pract Oncol 5: 136-147, 2008.

2. Wicki A and Christofori G: The potential role of podoplanin in tumour invasion. Br J Cancer 96: 1-5, 2007.

3. Ramirez MI, Millien G, Hinds A, Cao Y, Seldin DC and Williams MC: T1alpha, a lung type I cell differentiation gene, is required for normal lung cell proliferation and alveolus formation at birth. Dev Biol 256: 61-72, 2003.

4. Schacht V, Ramirez MI, Hong YK, et al: T1alpha/podoplanin deficiency disrupts normal lymphatic vasculature formation and causes lymphedema. EMBO J 22: 3546-3556, 2003.

5. Breiteneder-Geleff S, Soleiman A, Kowalski H, et al: Angiosarcomas express mixed endothelial phenotypes of blood and lymphatic capillaries: podoplanin as a specific marker for lymphatic endothelium. Am J Pathol 154: 385-394, 1999.

6. Kato Y, Kaneko M, Sata M, Fujita N, Tsuruo T and Osawa M: Enhanced expression of Aggrus (T1alpha/podoplanin), a plateletaggregation-inducing factor in lung squamous cell carcinoma. Tumour Biol 26: 195-200, 2005.

7. Martin-Villar E, Scholl FG, Gamallo C, et al: Characterization of human PA2.26 antigen (T1alpha-2, podoplanin), a small membrane mucin induced in oral squamous cell carcinomas. Int J Cancer 113: 899-910, 2005.

8. Schacht V, Dadras SS, Johnson LA, Jackson DG, Hong YK and Detmar M: Up-regulation of the lymphatic marker podoplanin, a mucin-type transmembrane glycoprotein, in human squamous cell carcinomas and germ cell tumors. Am J Pathol 166: 913-921, 2005.

9. Shibahara J, Kashima T, Kikuchi Y, Kunita A and Fukayama M: Podoplanin is expressed in subsets of tumors of the central nervous system. Virchows Arch 448: 493-499, 2006.

10. Wicki A, Lehembre F, Wick N, Hantusch B, Kerjaschki D and Christofori G: Tumor invasion in the absence of epithelialmesenchymal transition: podoplanin-mediated remodeling of the actin cytoskeleton. Cancer Cell 9: 261-272, 2006.

11. Kimura $\mathbf{N}$ and Kimura I: Podoplanin as a marker for mesothelioma. Pathol Int 55: 83-86, 2005.

12. Ordonez NG: Podoplanin: a novel diagnostic immunohistochemical marker. Adv Anat Pathol 13: 83-88, 2006.

13. Hinterberger M, Reineke T, Storz M, Weder W, Vogt P and Moch H: D2-40 and calretinin - a tissue microarray analysis of 341 malignant mesotheliomas with emphasis on sarcomatoid differentiation. Mod Pathol 20: 248-255, 2007.

14. Padgett DM, Cathro HP, Wick MR and Mills SE: Podoplanin is a better immunohistochemical marker for sarcomatoid mesothelioma than calretinin. Am J Surg Pathol 32: 123-127, 2008.

15. Mimura T, Ito A, Sakuma T, et al: Novel marker D2-40, combined with calretinin, CEA, and TTF-1: an optimal set of immunodiagnostic markers for pleural mesothelioma. Cancer 109: 933-938, 2007.

16. Marchevsky AM: Application of immunohistochemistry to the diagnosis of malignant mesothelioma. Arch Pathol Lab Med 132: 397-401, 2008. 
17. Kawaguchi H, El-Naggar AK, Papadimitrakopoulou V, et al: Podoplanin: a novel marker for oral cancer risk in patients with oral premalignancy. J Clin Oncol 26: 354-360, 2008.

18. Horiguchi A, Ito K, Sumitomo M, Kimura F, Asano T and Hayakawa M: Intratumoral lymphatics and lymphatic invasion are associated with tumor aggressiveness and poor prognosis in renal cell carcinoma. Urology 71: 928-932, 2008.

19. Mishima K, Kato Y, Kaneko MK, Nishikawa R, Hirose T and Matsutani M: Increased expression of podoplanin in malignant astrocytic tumors as a novel molecular marker of malignant progression. Acta Neuropathol 111: 483-488, 2006.

20. Martin-Villar E, Megias D, Castel S, Yurrita MM, Vilaro S and Quintanilla M: Podoplanin binds ERM proteins to activate RhoA and promote epithelial-mesenchymal transition. J Cell Sci 119: 4541-4553, 2006.

21. Kunita A, Kashima TG, Morishita Y, et al: The platelet aggregation-inducing factor aggrus/podoplanin promotes pulmonary metastasis. Am J Pathol 170: 1337-1347, 2007.

22. Deroanne CF, Hamelryckx D, Ho TT, et al: Cdc42 downregulates MMP-1 expression by inhibiting the ERK1/2 pathway. J Cell Sci 118: 1173-1183, 2005

23. Zeng ZS, Shu WP, Cohen AM and Guillem JG: Matrix metalloproteinase-7 expression in colorectal cancer liver metastases: evidence for involvement of MMP-7 activation in human cancer metastases. Clin Cancer Res 8: 144-148, 2002.

24. Tutton MG, George ML, Eccles SA, Burton S, Swift RI and Abulafi AM: Use of plasma MMP-2 and MMP-9 levels as a surrogate for tumour expression in colorectal cancer patients. Int J Cancer 107: 541-550, 2003.

25. Klominek J, Baskin B and Hauzenberger D: Platelet-derived growth factor (PDGF) BB acts as a chemoattractant for human malignant mesothelioma cells via PDGF receptor beta-integrin alpha3beta1 interaction. Clin Exp Metastasis 16: 529-539, 1998.

26. Mueller L, Goumas FA, Himpel S, Brilloff S, Rogiers X and Broering DC: Imatinib mesylate inhibits proliferation and modulates cytokine expression of human cancer-associated stromal fibroblasts from colorectal metastases. Cancer Lett 250 329-338, 2007
27. Faried A, Faried LS, Kimura H, et al: RhoA and RhoC proteins promote both cell proliferation and cell invasion of human oesophageal squamous cell carcinoma cell lines in vitro and in vivo. Eur J Cancer 42: 1455-1465, 2006.

28. Spugnini EP, Cardillo I, Verdina A, et al: Piroxicam and cisplatin in a mouse model of peritoneal mesothelioma. Clin Cancer Res 12: 6133-6143, 2006.

29. Zhong J, Gencay MM, Bubendorf L, et al: ERK1/2 and p38 MAP kinase control MMP-2, MT1-MMP, and TIMP action and affect cell migration: a comparison between mesothelioma and mesothelial cells. J Cell Physiol 207: 540-552, 2006.

30. Gorelik E, Bere WW and Herberman RB: Role of NK cells in the antimetastatic effect of anticoagulant drugs. Int J Cancer 33: 87-94, 1984.

31. Scholl FG, Gamallo C, Vilaró S and Quintanilla M: Identification of PA2.26 antigen as a novel cell-surface mucin-type glycoprotein that induces plasma membrane extensions and increased motility in keratinocytes. J Cell Sci 112: 4601-4613, 1999.

32. Suzuki-Inoue K, Kato Y, Inoue O, et al: Involvement of the snake toxin receptor CLEC-2, in podoplanin-mediated platelet activation, by cancer cells. J Biol Chem 282: 25993-26001, 2007.

33. Edwards JG, McLaren J, Jones JL, Waller DA and O'Byrne KJ: Matrix metalloproteinases 2 and 9 (gelatinases A and B) expression in malignant mesothelioma and benign pleura. Br J Cancer 88: 1553-1559, 2003.

34. Baker SJ and Reddy EP: Modulation of life and death by the TNF receptor superfamily. Oncogene 17: 3261-3270, 1998.

35. Wada T and Penninger JM: Mitogen-activated protein kinases in apoptosis regulation. Oncogene 23: 2838-2849, 2004.

36. Lee S, Yoon S and Kim DH: A high nuclear basal level of ERK2 phosphorylation contributes to the resistance of cisplatin-resistant human ovarian cancer cells. Gynecol Oncol 104: 338-344, 2007. 